\title{
Finite sets of operations sufficient to construct any fullerene from $C_{20}$
}

\author{
Victor M. Buchstaber · Nikolay Yu. Erokhovets
}

Received: date / Accepted: date

\begin{abstract}
We study the well-known problem of combinatorial classification of fullerenes. By a (mathematical) fullerene we mean a convex simple three dimensional polytope with all facets pentagons and hexagons. We analyse approaches of construction of arbitrary fullerene from the dodecahedron (a fullerene $C_{20}$ ). A growth operation is a combinatorial operation that substitutes the patch with more facets and the same boundary for the patch on the surface of a simple polytope to produce a new simple polytope. It is known that an infinite set of different growth operations transforming fullerenes into fullerenes is needed to construct any fullerene from the dodecahedron. We prove that if we allow a polytope to contain one exceptional facet, which is a quadrangle or a heptagon, then a finite set of growth operation is sufficient. We analyze pairs of objects: a finite set of operations, and a family of acceptable polytopes containing fullerenes such that any polytope of the family can be obtained from the dodecahedron by a sequence of operations from the corresponding set. We describe explicitly three such pairs. First two pairs contain seven operations, and the last - eleven operations. Each of these operations corresponds to a finite set of growth operations and is a composition of edge- and two edges-truncations.
\end{abstract}

Keywords Fullerenes - Growth operations · Finite sets · Polytopes · Edge-truncations · Dodecahedron

Mathematics Subject Classification (2000) MSC 52B10 . MSC 90C57

This work is supported by the Russian Science Foundation under grant 14-11-00414.

Victor M. Buchstaber · Nikolay Yu. Erokhovets

Steklov Mathematical Institute of Russuian Academy of Sciences, Gubkina str. 8, 119991, Moscow, Russia

Tel.: +7-495-9848141*3960

Fax: +7-495-9848139

E-mail: buchstab@mi.ras.ru, erochovetsn@hotmail.com

\section{Preface}

For any mathematical fullerene the Euler's formula implies that the number of pentagons is twelve. Nowadays the Euler formula and this consequence are known far beyond the mathematics and the gratitude to Euler is expressed in the Nobel lecture of one of the laureates for discovery of fullerenes. The natural question arises: What other restrictions on physical fullerenes follow from the combinatorics of mathematical counterparts. One of these restrictions is a mathematical result that the number of hexagons can not be equal to one. For centuries mathematics collected many characteristics to study the combinatorics of convex bodies: edge graph, Hamilton paths and cycles, belts of facets, regular colorings, perfect matchings (corresponding to Kekulé structures in chemistry), etc. Some of these notions found applications in the well-known problem: To obtain a fullerene with given number of hexagons as a result of reconstruction of a fullerene with less hexagons. In our article we present new results in this direction.

Our study was inspired by the encouraging book E.A. Lord, A.L. Mackay, S. Ranganathan. New Geometries for New Materials. Cambridge University Press, 2006 [1]. We submit this article to the Alan L Mackay special issue as a sign of our deep respect to this outstanding scientist and with the hope that our results will find applications in problems of physics and chemistry of fullerenes.

\section{Introduction}

We will use notions and results of the polytope theory following [2,3].

Definition 1 By a (mathematical) fullerene we mean a simple convex 3-polytope with all facets pentagons and hexagons. 


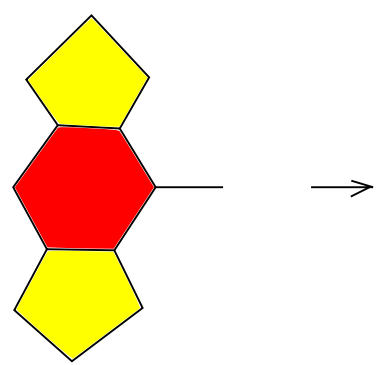

Fig. 1 Endo-Kroto operation

Simple means that any vertex belongs to exactly three facets. An IPR-fullerene is a fullerene without adjacent pentagons.

This is a mathematical model for a fullerene - a carbon molecule synthesized in 1985 (Nobel prize 1996 in chemistry to Robert Curl, Harold Kroto, and Richard Smalley [4, 5,6]). Since discovery of fullerenes they were studied extensively from the mathematical point of view. As mentioned above the Euler formula implies that any fullerene contains exactly $p_{5}=12$ pentagons, while it is not hard to prove that the number $p_{6}$ of hexagons can be arbitrary nonnegative integer except for one. There is only one combinatorial type of fullerenes with $p_{6}=0$ (the dodecahedron $C_{20}$ ), while from the work by W. Thurston [7] it follows that for large values of $p_{6}$ the number of combinatorial types of fullerenes growth like $p_{6}^{9}$. There are effective algorhythms of combinatorial enumeration of fullerenes on supercomputers by $\mathrm{G}$. Brinkmann and A. Dress [8] and later to G. Brinkmann, J. Goedgebeur, and B.D. McKay [9]. For example, for $p_{6}=75$ there are 46088157 combinatorial types of fullerenes (see [10]). Thus, the important problem is to define and study different structures and operations on the set of all combinatorial types of fullerenes. The well-known problem [9, 11 , 12, 13, 14, 15, 16 is to find a simple set of operations sufficient to construct arbitrary fullerene from the dodecahedron $C_{20}$.

Definition 2 A patch is a disk bounded by a simple edgecycle on the boundary of a simple 3-polytope. Any patch is topologically a disk and the complement to a patch is also a disk. A growth operation is a combinatorial operation that gives a new 3-polytope $Q$ from a simple 3-polytope $P$ by substituting a new patch with the same boundary and more facets for the patch on the boundary of $P$.

The Endo-Kroto operation [17] (Fig. 11) is the simplest example of a growth operation that transforms a fullerene into a fullerene. It increases $p_{6}$ by one. It was proved in [15] that there is no finite set of growth operations transforming fullerenes into fullerenes sufficient to construct arbitrary fullerene from the dodecahedron. In [12] the example of an infinite set was found.

We construct finite sets of growth operations and corresponding families of simple 3-polytopes extending the set

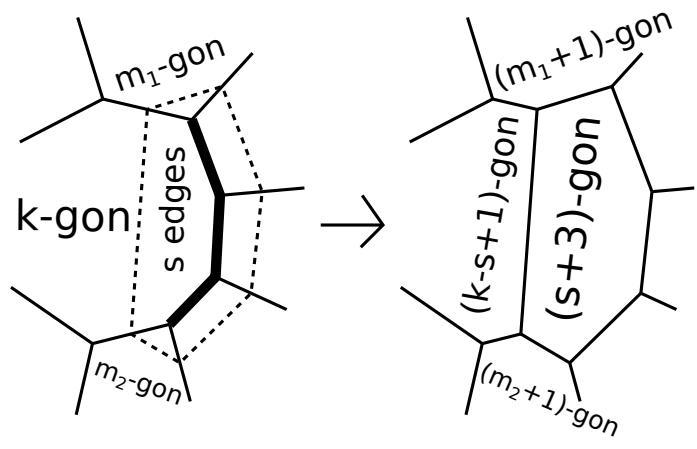

Fig. $2(s, k)$-truncation

of fullerenes such that any polytope of the family can be combinatorially constructed from the dodecahedron by a sequence of these operations and all intermediate polytopes belong to the family. The less polytopes the extended family contains the more operations we need. All of the operations we construct are composition of edge- and two edgestruncations, see Definition 3 .

\section{Main tools}

\section{$3.1(s, k)$-truncations}

Definition 3 Let $F$ be a $k$-gonal facet of a simple 3-polytope. Choose $s$ consequent edges of $F$, rotate the supporting hyperplane of $F$ around the axis passing through the midpoints of adjacent two edges (one on each side), and take the corresponding hyperplane truncation (see Fig. 2).

We call this operation an $(s, k)$-truncation. If the facets intersecting $F$ by the edges adjacent to truncated sequence of edges are $m_{1}$ - and $m_{2}$-gons, then we also call the operation an $\left(s, k ; m_{1}, m_{2}\right)$-truncation. The reflexion of the space $\mathbb{R}^{3}$ changes $m_{1}$ - and $m_{2}$-gons, so we will not distinguish between $\left(s, k ; m_{1}, m_{2}\right)$ - and $\left(s, k ; m_{2}, m_{1}\right)$-truncations. For $s=1$ the truncated edge belongs to two facets, so we denote these operation simply as $\left(1 ; m_{1}, m_{2}\right)$-truncation.

Example 1 1. A vertex-truncation is a $(0, k)$-truncation.

2. An edge-truncation is a $(1, k)$-truncation.

3. The Endo-Kroto operation is a $(2,6)$-truncation.

4. (2,6)-truncation is the only $(s, k)$-truncation that transforms a fullerene to a fullerene.

Remark 1 For $s=0$ and $s=k-2$ we have the vertex-truncation, which can be considered as the substitution of the corresponding patch for the three facets containing the vertex; hence it is a growth operation. Let $0<s<k-2$ and the $m_{1}$-gon and the $m_{2}$-gon do not intersect. Then the union of a $k$-gon and these facets form a patch, and after the $(s, k)$ truncation we have the new patch with combinatorially the same boundary (see Fig. 3 on the left). Thus we have a 

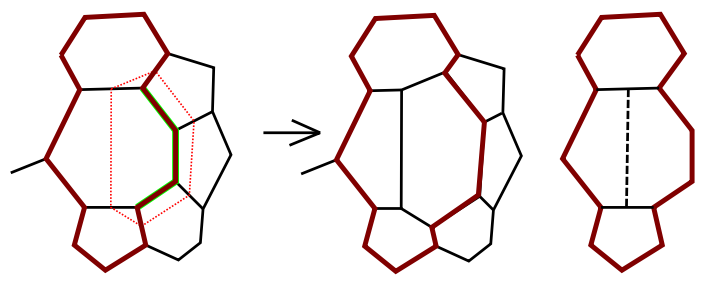

Fig. $3(s, k)$-truncation as a growth operation

growth operation, which we denote by the scheme on Fig. 3 on the right.

For $s=1$ as mentioned above the edge-truncation can be considered as a $(1, k)$-truncation and a $\left(1, k^{\prime}\right)$-truncation for two facets containing the truncated edge. This gives two different patches, which differ by one facet.

Theorem 1 (see [18,19]) A 3-polytope is simple if and only if it is combinatorially equivalent to a polytope obtained from the tetrahedron by a sequence of vertex-, edge- and $(2, k)$-truncations.

This result was used in works of famous crystallographer E.S. Fedorov.

\section{$3.2 k$-belts and flag polytopes}

We will denote the combinatorial equivalence of polytopes $P$ and $Q$ by $P \simeq Q$. The polytope combinatorially equivalent to the tetrahedron we call simplex and denote $\Delta^{3}$.

Definition 4 A cyclic sequence of $k$ facets $\left(F_{i_{1}}, \ldots, F_{i_{k}}\right), F_{i_{k+1}}=$ $F_{i_{1}}$, is called a $k$-belt if $F_{i_{1}} \cap \ldots \cap F_{i_{k}}=\varnothing$, and $F_{i_{p}} \cap F_{i_{q}} \neq \varnothing$ if and only if $p-q= \pm 1 \bmod k$.

A simple 3-polytope is called flag, if it is not a simplex and has no 3-belts. Equivalently, any set of pairwise adjacent facets has nonempty intersection.

It is easy to prove that if $P$ is a flag 3-polytope, then the $(s, k)$-truncation transforms it to a flag polytope if and only if $0<s<k-2$.

For the structure of $k$-belts on simple 3-polytopes with at most hexagonal facets see [20]. Related results on cyclic $k$-edge cuts see in [21,22,23,24, 25].

In [26] the analog of Theorem 1 for flag polytopes was proved.

Theorem 2 A simple 3-polytope is flag if and only if it is combinatorially equivalent to a polytope obtained from the cube by a sequence of edge- and $(2, k)$-truncations, $k \geq 6$.

For us this result is important, since any fullerene is a flag polytope. This follows from [21]. We will also need a more general result.

Theorem $3([27,28])$ Let $P$ be simple 3-polytope with $p_{3}=$ $0, p_{4} \leq 2, p_{7} \leq 1$, and $p_{k}=0, k \geq 8$. Then it has no 3-belts. In particular, it is a flag polytope.
Also we will need the following results. In the case of fullerenes it follows from [22].

Theorem $4([27,28])$ Let $P$ be a simple polytope with all facets pentagons and hexagons with at most one exceptional facet $F$ being a quadrangle or a heptagon.

1. If $P$ has no quadrangles, then $P$ has no 4-belts.

2. If $P$ has a quadrangle $F$, then there is exactly one 4-belt. It surrounds $F$.

Our main Theorems 6, 9 and 10 imply the following result.

Theorem 5 Any fullerene can be obtained from the dodecahedron by a sequence of $p_{6}$ edge-and only $(2,6)$ - and $(2,7)$ truncations.

\subsection{Straightening along an edge}

It is important that in some cases there is an operation inverse to the $(s, k)$-truncation.

Definition 5 For some edges $F_{p} \cap F_{q}$ of a simple 3-polytope $P$ there is an operation of straightening along the edge (see Fig. 4).

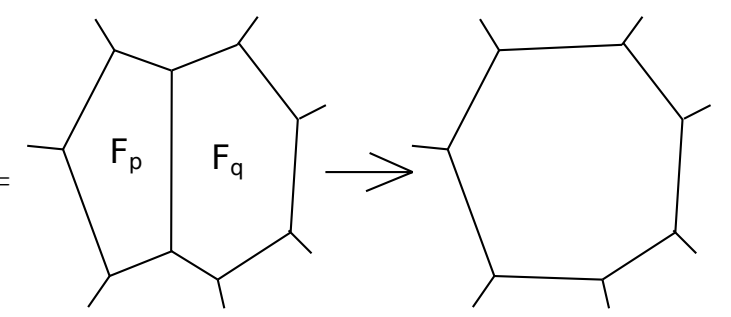

Fig. 4 Straightening along the edge

We will need the following result.

Lemma 1 ([26, 28]) For $P \simeq \Delta^{3}$ no straightening operations are defined. Let $P \neq \Delta^{3}$ be a simple polytope. For an edge $F_{i} \cap F_{j}$ the operation of straightening is defined if and only if there is no 3-belt $\left(F_{i}, F_{j}, F_{k}\right)$ for some $F_{k}$.

Remark 2 We see that $(s, k)$-truncation is a combinatorial operation and is always defined. It is easy to show that the straightening along the edge is a combinatorially inverse operation. Lemma 1 gives the criterion when it is defined.

Corollary 1 A polytope $P$ is flag if and only if straightening along any its edge is defined.

Theorem 3 implies the following result.

Corollary 2 Let $P$ be a fullerene, or more generally, a simple polytope with $p_{3}=0, p_{4} \leq 2, p_{7} \leq 1$, and $p_{k}=0$ for $k \geq 8$. Then straightening is defined along any edge. 


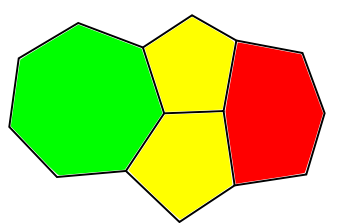

a)

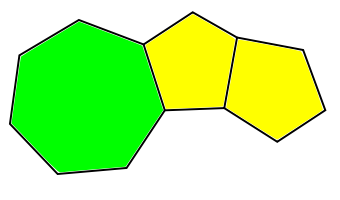

b)
Fig. 5 Fragments on a polytope in $\mathscr{F}_{1}$

In fact we will need a special case that the straightenings we use are defined. This can be also proved using the following remarkable result.

Lemma 2 ([29], Case 2 in Subsection 2.5) Let the sphere $S^{2}$ be glued edge-to-edge from pentagons, hexagons, and one $k$-gon with $3 \leqslant k \leqslant 7$, such that the obtained graph is 3-valent. Then this partition is combinatorially equivalent to the boundary of a simple 3-polytope.

Corollary 3 If a straightening along the edge of a simple 3polytope with all facets pentagons, hexagons and one facet quadrangle or a heptagon gives a simple partition of the sphere $S^{2}$ with the same properties, then the straightening is well-defined.

\section{Construction of fullerenes by truncations}

\subsection{Seven truncations}

Definition 6 Let $\mathscr{F}_{-1}$ be the set of combinatorial simple 3polytopes with all facets pentagons and hexagons except for one facet quadrangle. The Euler formula implies that any polytope in $\mathscr{F}_{-1}$ has $p_{5}=10$.

Let $\mathscr{F}$ be the set of all fullerenes, and $\mathscr{F}^{I P R}$ be the set of all IPR-fullerenes.

Let $\mathscr{F}_{1}$ be the set of simple 3-polytopes with all facets pentagons and hexagons except for one facet heptagon adjacent to a pentagon such that either there are two pentagons with the common edge intersecting the heptagon and a hexagon (Fig. 5a), or for any two adjacent pentagons exactly one of them is adjacent to the heptagon (Fig. 5b). Any polytope in $\mathscr{F}_{1}$ has $p_{5}=13$. Let $\mathscr{F}_{1}^{I P R}$ be the set of polytopes in $\mathscr{F}_{1}$ without adjacent pentagons. Set $\mathscr{F}_{s}=\mathscr{F}_{-1} \sqcup \mathscr{F} \sqcup \mathscr{F}_{1}$.

Theorem $6\left([27,[28])\right.$ Any polytope in $\mathscr{F}_{s}$ can be obtained from the dodecahedron by a sequence of $p_{6}+2 p_{7}-p_{4}$ truncations: $\quad(1 ; 4,5)-, \quad(1 ; 5,5)-, \quad(2,6 ; 4,5)-, \quad(2,6 ; 5,5)-$, $(2,6 ; 5,6)-,(2,7 ; 5,5)-$, and $(2,7 ; 5,6)-$, in such a way that intermediate polytopes belong to $\mathscr{F}_{s}$. Moreover (see Fig. 6),

1. any polytope in $\mathscr{F}_{-1}$ can be obtained by a $(1 ; 5,5)-$ or $(1 ; 4,5)$-truncation from a polytope in $\mathscr{F}$ or $\mathscr{F}_{-1}$ respectively;

2. any polytope in $\mathscr{F}_{1}$ can be obtained by a $(2,6 ; 5,6)$ - or $(2,7 ; 5,6)$-truncation from a polytope in $\mathscr{F}$ or $\mathscr{F}_{1}$ respectively;

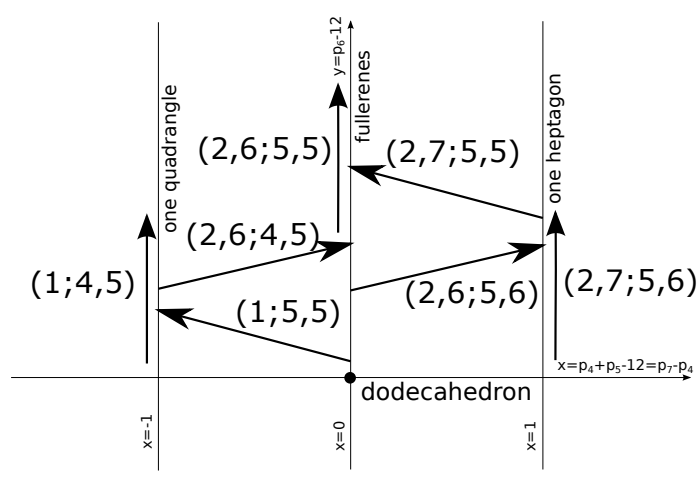

Fig. 6 Scheme of the truncation operations

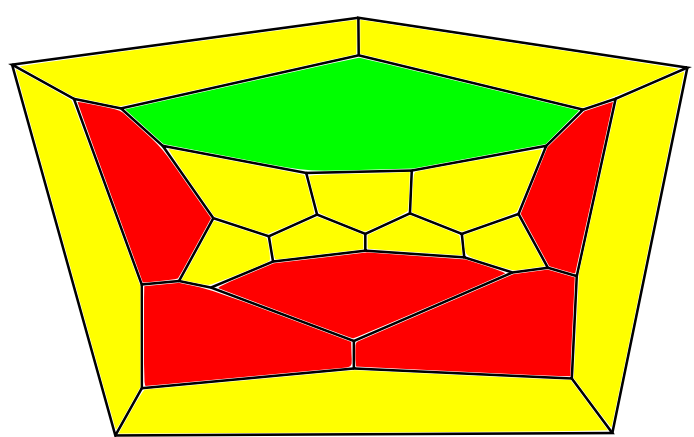

Fig. 7 Polytope not in $\mathscr{F}_{1}$ that can not be obtained as a result of our seven truncations

3. polytope in $\mathscr{F}$ can be obtained by a $(2,6 ; 5,5)$-, $(2,6 ; 4,5)$-, or $(2,7 ; 5,5)$-truncation from a polytope in $\mathscr{F}, \mathscr{F}_{-1}$ or $\mathscr{F}_{1}$ respectively.

Remark 3 Fig. 7 together with Lemma 2 shows a simple polytope $P \notin \mathscr{F}_{1}$ with all facets pentagons, hexagons, and only one heptagon, which is adjacent to a pentagon. This polytope can not be obtained by the above seven truncations from a polytope $Q$ in the same class. Indeed, $(1 ; 4,5)-,(1 ; 5,5)-$, and $(2,6 ; 4,5)$-truncation are not allowed, since $P$ has no quadrangles, and $Q$ can not contain a quadrangle and a heptagon simultaneously. $Q$ should be obtained from $P$ by a straightening along some edge. This edge can belong either to two pentagons, or to a hexagon and a pentagon. Common edge of two pentagons in $P$ either intersects two pentagons, or a pentagon and a hexagon, or a pentagon and the heptagon. This corresponds to $(2,6 ; 4,4)$-, $(2,6 ; 4,5)$-, or $(2,6 ; 4,6)$-truncations; hence $(2,6 ; 5,5)$ - and $(2,6 ; 5,6)$-truncations are not allowed. Common edge of a pentagon and a hexagon in $P$ either intersects two pentagons, or a pentagon and a hexagon, or two hexagons, or a pentagon and the heptagon. This corresponds to $(2,7 ; 4,4)$-, $(2,7 ; 4,5)-,(2,7 ; 5,5)-$, or $(2,7 ; 5,7)$-truncations, hence $(2,7 ; 5,6)$-truncation is also not allowed. In the case of $(2,7 ; 5,5)$-truncation $Q$ contains two heptagons; hence this operation is also not allowed. 


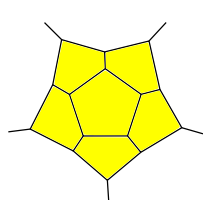

a)

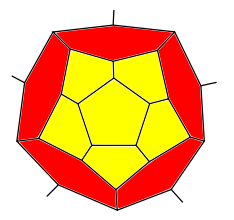

b)

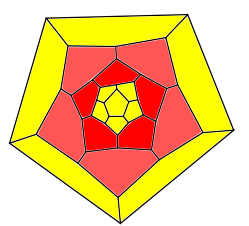

c)
Fig. 8 Construction of the first family of fullerenes
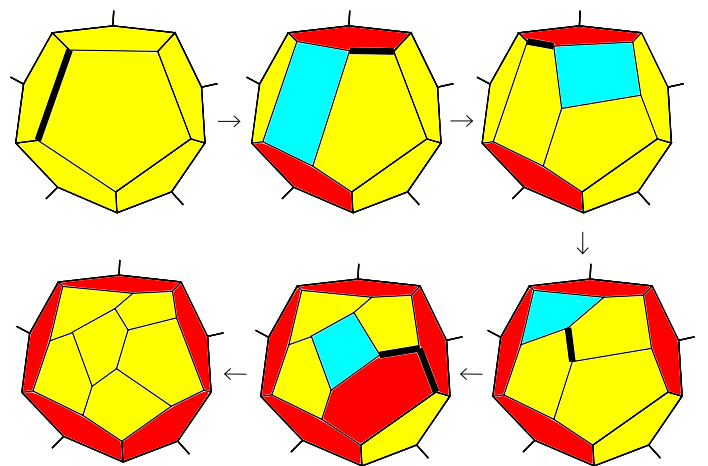

Fig. 9 Insertion of a belt as a sequence of truncations

\subsection{Getting rid of quadrangles}

It turns out that in Theorem 6 we can get rid of quadrangles.

Two families of fullerenes

Definition 7 1. Take the dodecahedral cap $C_{1}$, drawn on Fig. 8a).

2. Add a 5-belt of hexagons around the patch (Fig. 8b). The boundary of the new patch is combinatorially the same.

3. After $k$ steps add the same cap again to obtain a fullerene $D_{5 k}$ (The case $k=2$ see on Fig. 8c).

Fullerenes $D_{5 k}$ for $k \geqslant 1$ are known as $(5,0)$-nanotubes.

The insertion of a belt can be realized as a sequence of $(1 ; 4,5)-,(1 ; 5,5)-$, and $(2,6 ; 4,5)$-truncations, see Fig. 9

Proposition 1 ([26, 28] $)$ A fullerene is combinatorially equivalent to $D_{5 k}$ for $k \geqslant 0$ if and only if it contains the cap $C_{1}$.

The significance of the family $D_{5 k}$ is described by the following theorem, which follows directly from [23] or [24].

Theorem $7([\mathbf{2 7}, \mathbf{2 8} \mid)$ Let $P$ be a fullerene. Then the following statements hold.

I. Any pentagonal facet is surrounded by a 5-belt. There are

12 belts of this type.

II. If there is a 5-belt not surrounding a pentagon, then

1. it consists only of hexagons;

2. the fullerene is combinatorially equivalent to the polytope $D_{5 k}, k \geqslant 1$.

3. the number of 5-belts is $12+k$.

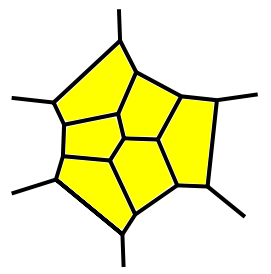

a)

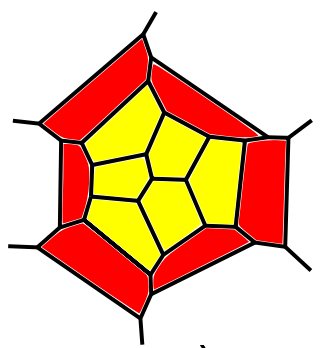

C)

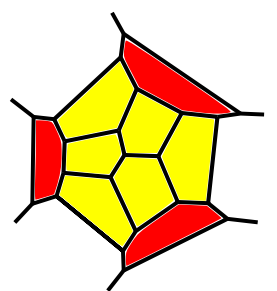

b)

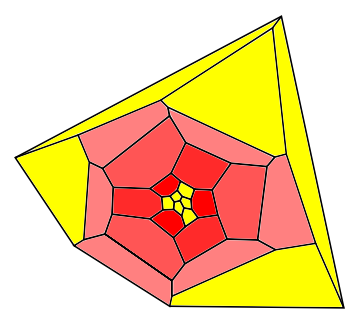

d)
Fig. 10 Construction of the second family of fullerenes

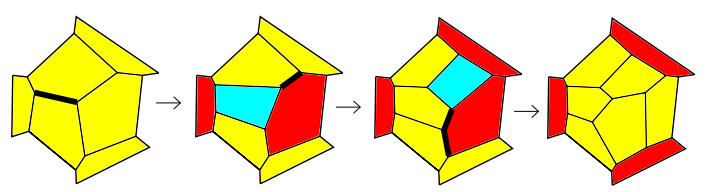

Fig. 11 Addition of three hexagons as a sequence of truncations

Definition 8 1. Take the dodecahedral cap $C_{2}$ drawn on Fig. 10a).

2. Add 3 hexagons adjacent to facets with single edge on the boundary (Fig. 10 b). The boundary of the patch remains combinatorially the same.

3. After $k$ steps $(k=2$ see on Fig. 10 c) add the same cap again to obtain a fullerene $F_{3 k}$ (The case $k=5$ see on Fig. 10d).

Fullerenes $F_{3 k}$ for $k \geqslant 2$ are also known as $(3,3)$-nanotubes.

The addition of three hexagons can be realized as a sequence of $(1 ; 4,5)-,(1 ; 5,5)$-, and $(2,6 ; 4,5)$-truncations, see Fig. 11

Theorem 8 ([26]) A fullerene is combinatorially equivalent to $F_{3 k}$ for $k \geqslant 0$ if and only if it contains the cap $C_{2}$.

Proof First introduce some notions.

Definition 9 Let $P$ be a simple polytope. A $k$-loop is a cyclic sequence $\left(F_{i_{1}}, \ldots, F_{i_{k}}\right), F_{i_{k+1}}=F_{i_{1}}$, of facets, such that $F_{i_{1}} \cap$ $F_{i_{2}}, \ldots, F_{i_{k-1}} \cap F_{i_{k}}, F_{i_{k}} \cap F_{i_{1}}$ are edges. A $k$-loop is called simple, if its facets are pairwise different. A simple edge-cycle $\gamma$ borders a $k$-loop $\mathscr{L}$ if $\mathscr{L}$ is the set of facets that appears when we walk along $\gamma$ in one of the two connected components of $(\partial P) \backslash \gamma$. We say that an $l_{1}$-loop $\mathscr{L}_{1}$ borders an $l_{2}$-loop $\mathscr{L}_{2}$ (along $\gamma$ ), if they border the same edge-cycle $\gamma$. 


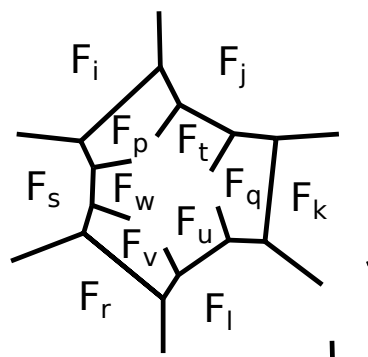

a)

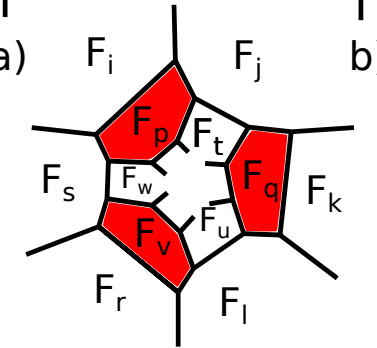

c)

Fig. 12 Fragments on the fullerene

Lemma 3 Let $P$ be a fullerene, and a 6-loop $\mathscr{L}_{1}=\left(F_{p}, F_{t}, F_{q}, F_{u}, F_{v}, F_{w}\right)$ border a simple 6-loop $\mathscr{L}_{2}=$ $\left(F_{i}, F_{j}, F_{k}, F_{l}, F_{r}, F_{s}\right)$ with $(1,3,1,3,1,3)$ edges on the boundary, as drawn on Fig. 12 a). Then $\mathscr{L}_{1}$ is simple and either forms the fragment on Fig. 12 b), or $F_{p}, F_{q}, F_{v}$ are hexagons and the 6-loop $\mathscr{L}_{3}=\left(F_{j}, F_{q}, F_{l}, F_{v}, F_{s}, F_{p}\right)$ is simple and has $(1,3,1,3,1,3)$ edges on the boundary component intersecting $F_{t}$.

Proof Let $\mathscr{L}_{1}$ and $\mathscr{L}_{2}$ border the same simple edge-cycle $\gamma$.

We have $F_{p} \neq F_{q}$, since they intersect $F_{j}$ by different edges. Also $F_{p} \cap F_{q}=\varnothing$, else $F_{p} \cap F_{q} \cap F_{j}$ is a vertex and $F_{j}$ has only 2 edges in $\gamma$, but not 3 . Similarly $F_{p} \cap F_{v}=\varnothing$. Then $F_{p} \neq F_{u}$, else $F_{p} \cap F_{q} \neq \varnothing$. We have $F_{t} \neq F_{u}$, else $F_{q}$ is a quadrangle. By the symmetry we obtain that the 6-loop $\mathscr{L}_{1}$ is simple and $F_{p} \cap F_{q}=F_{q} \cap F_{v}=F_{v} \cap F_{q}=\varnothing$.

Let $F_{t} \cap F_{w} \neq \varnothing$. Then $F_{p} \cap F_{t} \cap F_{w}$ is a vertex and $F_{p}$ is a pentagon. We have a simple 5-loop $\mathscr{L}_{5}=\left(F_{t}, F_{q}, F_{u}, F_{v}, F_{w}\right)$. It is not a 5-belt, else by Theorem 7 it should either surround a pentagon, and in this case $F_{u}$ is a quadrangle, or consist of hexagons and have $(2,2,2,2,2)$ edges on each boundary component. But $F_{u}$ has a single edge on the boundary component. We have $F_{t} \cap F_{v} \neq \varnothing$, else $F_{t} \cap F_{w} \cap F_{v}$ is a vertex and $F_{w}$ is a quadrangle. Similarly $F_{q} \cap F_{w}=\varnothing$. Also recall that $F_{q} \cap F_{v}=\varnothing$. Then either $F_{t} \cap F_{u} \neq \varnothing$, or $F_{u} \cap F_{w} \neq$ $\varnothing$. In the first case $\left(F_{t}, F_{u}, F_{v}, F_{w}\right)$ is a simple 4-loop with $F_{t} \cap F_{v}=\varnothing$. Since $P$ has no 4-belts we have $F_{u} \cap F_{w} \neq \varnothing$. Then $F_{u} \cap F_{w} \cap F_{v}, F_{u} \cap F_{w} \cap F_{t}$, and $F_{t} \cap F_{u} \cap F_{q}$ are vertices and we obtain Fig. 12 b). In the second case we obtain the same picture by the symmetry.

If $F_{t} \cap F_{w}=\varnothing$, then $F_{p}$ is a hexagon.

By the symmetry considering the facets $F_{q}$ and $F_{v}$ we obtain that either all of them are pentagons, and we obtain Fig.
$12 \mathrm{~b})$, or all of them are hexagons, and we obtain Fig. 12k). Since $F_{p} \cap F_{q}=F_{q} \cap F_{v}=F_{v} \cap F_{w}=\varnothing$, the boundary component of $\mathscr{L}_{3}$ intersecting $F_{t}$ is a simple edge-cycle. Since both sets $\left\{F_{j}, F_{l}, F_{s}\right\}$ and $\left\{F_{q}, F_{v}, F_{p}\right\}$ consist of pairwise different facets and these sets belong to different connected components with respect to $\gamma$, the 6-loop $\mathscr{L}_{3}$ is simple. It has $(1,3,1,3,1,3)$ edges on the boundary component intersecting $F_{t}$.

Let $P$ contain the fragment on Fig. 12b). Consider the 6-loop $\mathscr{L}=\left(F_{p}, F_{t}, F_{q}, F_{u}, F_{v}, F_{w}\right)$. The facets $\mathscr{L} \backslash\left\{F_{v}\right\}$ are pairwise different, since four of them surround the fifth. The same is for $\mathscr{L} \backslash\left\{F_{p}\right\}$ and $\mathscr{L} \backslash\left\{F_{q}\right\}$. Any two facets of $\mathscr{L}$ belong to one of these sets, therefore $\mathscr{L}$ is a simple loop. $F_{p} \cap F_{q}=\varnothing$, else $F_{p} \cap F_{q} \cap F_{t}$ is a vertex and $F_{t}$ is a quadrangle. Similarly $F_{q} \cap F_{v}=F_{v} \cap F_{p}=\varnothing$. Then $\partial \mathscr{L}$ is a simple edge-cycle, and $\mathscr{L}$ has $(1,3,1,3,1,3)$ edges on it. Applying Lemma 3 to $\mathscr{L}$ we obtain the proof of the theorem.

Remark 4 We also have proved that any fragment of the form $C_{2}$ is bounded by a simple edge-cycle, hence is a patch.

Construction of fullerenes without quadrangles on the steps

Theorem 9 Any polytope in $\mathscr{F} \sqcup \mathscr{F}_{1}$ can be combinatorially obtained from the dodecahedron by operations $A_{1}-A_{7}$ (see Fig. 13) such that all intermediate polytopes belong to $\mathscr{F} \sqcup \mathscr{F}_{1}$. Moreover,

1. any polytope in $\mathscr{F} \backslash \mathscr{F} I P R$ can be obtained from a polytope in $\mathscr{F}$ by one of operations $A_{1}-A_{4}$;

2. any polytope in $\mathscr{F} I P R$ can be obtained from a polytope in $\mathscr{F}_{1}$ by operation $A_{6}$

3. any polytope in $\mathscr{F}_{1}$ can be obtained by operations $A_{5}$ or $A_{7}$ from a polytope in $\mathscr{F}$ or $\mathscr{F}_{1}$ respectively;

4. operations $A_{1}-A_{3}$ are compositions of $(1 ; 4,5)$-, $(1 ; 5,5)-$, and $(2,6 ; 4,5)$-truncations;

5. operations $A_{4}, A_{5}, A_{6}, A_{7}$ are $(2,6 ; 5,5)$-, $(2,6 ; 5,6)$-, $(2,7 ; 5,5)-$, and $(2,7 ; 5,6)$-truncations respectively.

Proof We will need the following result.

Lemma 4 If a fullerene $P$ has two adjacent pentagons, then either it contains patch $C_{1}$, or patch $C_{2}$, or one of the patches drawn on Fig. 14

Proof Since any fullerene has no 3- and 4-belts, any fragment on a fullerene of the form $C_{1}, C_{2}, P_{1}$, or $P_{2}$ is bounded by a simple edge-cycle; hence it is a patch.

By assumption $P$ contains two adjacent pentagons $F_{i}$ and $F_{j}$. Consider the facets $F_{k}, F_{l}$ such that $F_{i} \cap F_{j} \cap F_{k}$ and $F_{i} \cap$ $F_{j} \cap F_{l}$ are vertices. If both facets $F_{k}, F_{l}$ are hexagons, then $P$ contains the fragment $P_{1}$. Else one of them is a pentagon. 


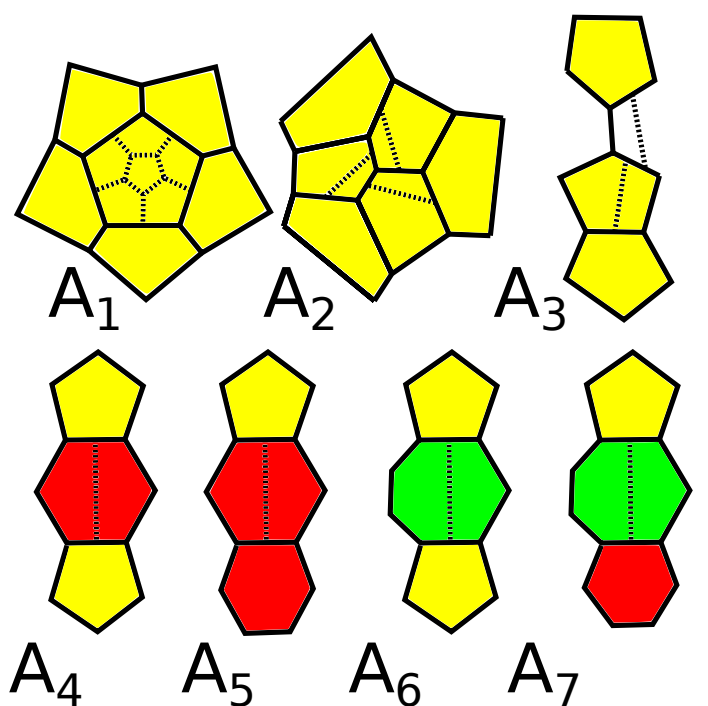

Fig. 13 Seven operations sufficient to construct any polytope in $\mathscr{F} \sqcup$ $\mathscr{F} 1$ from the dodecahedron. Dotted lines correspond to edges on the resulting polytope

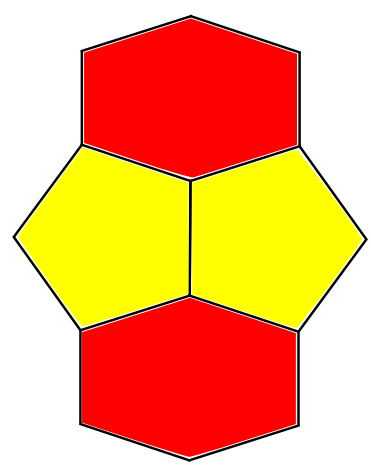

a)

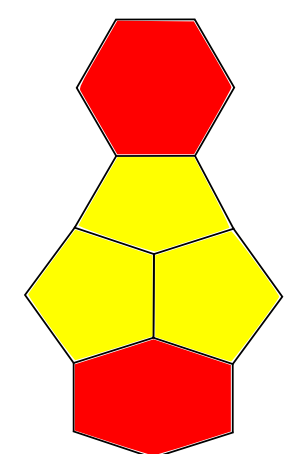

b)
Fig. 14 a) Patch $P_{1}$; b) Patch $P_{2}$

Without loss of generality let it be $F_{k}$. We obtain three pentagons with a common vertex, as drawn on Fig. 15]a).

If $F_{p}, F_{q}, F_{l}$ are pentagons, we obtain the fragment $C_{2}$.

Let two of them, say $F_{p}$ and $F_{q}$, be hexagons. Then either $F_{u}$ is a pentagon and we obtain the fragment $P_{1}$, or $F_{u}$ is a hexagon, and we obtain Fig. 15 b). If $F_{v}, F_{l}$, or $F_{w}$ is a hexagon, we obtain the fragment $P_{2}$. If all of them are pentagons, we obtain the fragment $C_{2}$.

Now let one of the facets $F_{p}, F_{q}, F_{l}$, say $F_{l}$, be a hexagon, and two others - pentagons, as drawn on Fig. $15 \mathrm{c}$ ). Then either $F_{u}$ is a hexagon and we obtain the fragment $P_{2}$, or it is a pentagon and we obtain the fragment $C_{1}$.

If a fullerene $P$ has two adjacent pentagons, then by Lemma 4 it contains one of the patches $C_{1}, C_{2}, P_{1}$ or $P_{2}$. If $P$ contains patch $C_{1}$ or $C_{2}$, then by Proposition 1 or Theorem 8 it is contained in the family $D_{5 k}$ or $F_{3 k}$ respectively, hence can be obtained from the dodecahedron by operations $A_{1}$ or $A_{2}$. Fig. 9

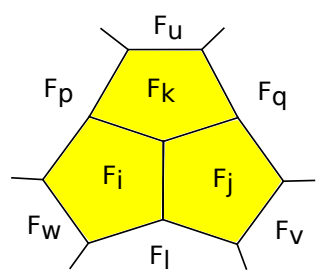

a)

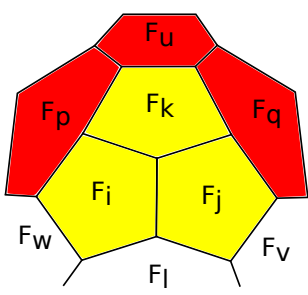

b)

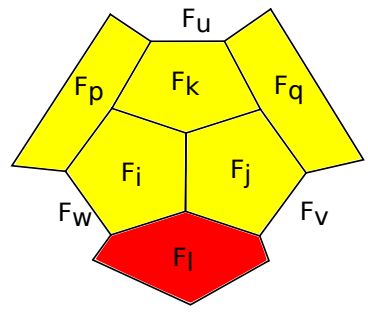

c)
Fig. 15 Fragments on a fullerene with adjacent pentagons

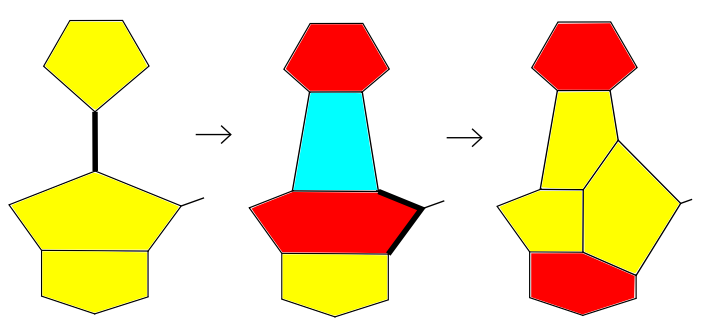

Fig. 16 Decomposition of operation $A_{3}$ into truncations

and Fig. 11 show how operations $A_{1}$ and $A_{2}$ are decomposed into $(1 ; 4,5)$-, $(1 ; 5,5)$-, and $(2,6 ; 4,5)$-truncations.

If $P$ contains patch $P_{1}$, then by Corollary $2 P$ is obtained from some other fullerene $Q$ by operation $A_{4}$, which is a $(2,6 ; 5,5)$-truncation.

Fig. 16 shows how operation $A_{3}$ decomposes into $(1 ; 5,5)$ and $(2,6 ; 4,5)$-truncations. If $P$ contains patch $P_{2}$, then applying the corresponding inverse operations by Corollary 2 we see that the fullerene is obtained from some other fullerene by operation $A_{3}$.

If a fullerene $P$ has no adjacent pentagons, then the proof of Theorem 9.12 in [28] implies that $P$ is obtained from a polytope in $\mathscr{F}_{1}$ by operation $A_{6}$, which is a $(2 ; 7 ; 5,5)$ truncation.

Theorem 6 implies that any polytope in $\mathscr{F}_{1}$ can be obtained by operations $A_{5}$ and $A_{7}$, from a fullerene or a polytope in $\mathscr{F}_{1}$ respectively. Here $A_{5}$ is a $(2,6 ; 5,6)$-truncation, and $A_{7}$ is a $(2,7 ; 5,6)$-truncation.

\subsection{Singular $I P R$-fullerenes}

Theorem 10 Any polytope in $\mathscr{F} \sqcup \mathscr{F}_{1}^{I P R}$ can be combinatorially obtained from the dodecahedron by operations $A_{1}-$ 


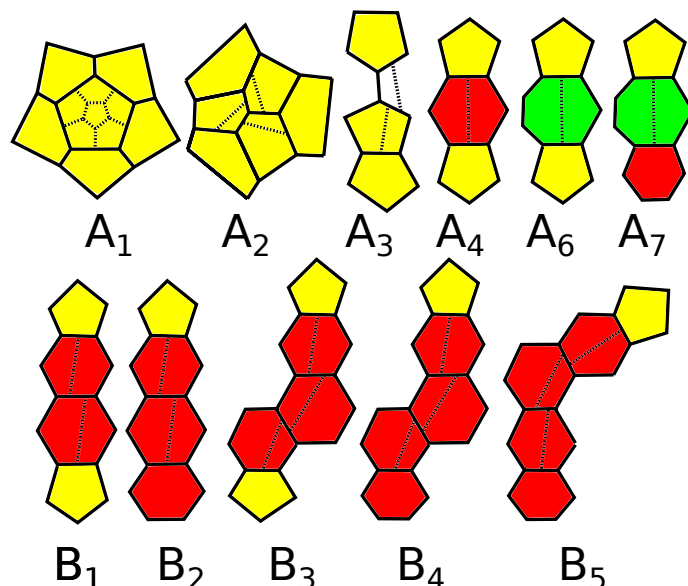

Fig. 17 Eleven operations sufficient to construct any polytope in $\mathscr{F} \sqcup$ $\mathscr{F}_{1}^{P R}$ from the dodecahedron. Dotted lines correspond to edges of the resulting polytope

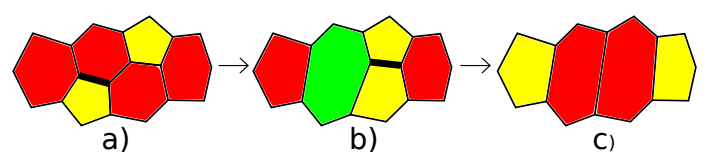

Fig. 18 a) A patch on the fullerene; b)-c) it's reductions

$A_{4}, A_{6}, A_{7}, B_{1}-B_{5}$ (Fig. 17) in such a way that all intermediate polytopes belong to $\mathscr{F} \sqcup \mathscr{F}_{1}^{I P R}$. Moreover,

1. any polytope in $\mathscr{F} \backslash \mathscr{F} I P R$ can be obtained from a polytope in $\mathscr{F}$ by one of operations $A_{1}-A_{4}$;

2. any polytope in $\mathscr{F}^{I P R}$ can be obtained by operations $B_{1}$ or $B_{3}$ from a polytope in $\mathscr{F}$, or by operation $A_{6}$ from a polytope in $\mathscr{F}_{1}^{I P R}$;

3. any polytope in $\mathscr{F}_{1}^{I P R}$ can be obtained by operations $B_{2}$, $B_{4}$, or $B_{5}$ from a polytope in $\mathscr{F}$, or by operation $A_{7}$ from a polytope in $\mathscr{F}_{1}^{I P R}$

4. operations $A_{1}-A_{3}$ are compositions of $(1 ; 4,5)$-, $(1 ; 5,5)-$, and $(2,6 ; 4,5)$-truncations;

5. operations $A_{4}, A_{6}, A_{7}$ are $(2,6 ; 5,5)-,(2,7 ; 5,5)$-, and $(2,7 ; 5,6)$-truncations respectively;

6. operations $B_{1}-B_{5}$ are compositions of $(2,6 ; 5,6)$-, $(2,7 ; 5,5)$-, and $(2,7 ; 5,6)$-truncations.

Proof The statement 1. is a part of Theorem 9

To prove the statement 2 . take an $I P R$-fullene $P$. Consider 3 cases.

Case 1. $P$ has an edge intersecting by vertices two pentagons. Since $P$ has no 3- and 4-belts, it contains the patch on Fig. 18 a). Then Fig. 18 b)-c) show how this patch can be reduced by straightening along edges. Then composition of the inverse operations gives operation $B_{1}$.

Case 2. $P$ has no edge intersecting two pentagons, but has a hexagon adjacent to two pentagons by opposite edges. The absence of 3- and 4-belts, and the structure of 5-belts given by Theorem 7 implies that $P$ contains the patch on a)

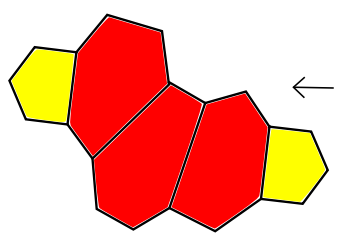

d)

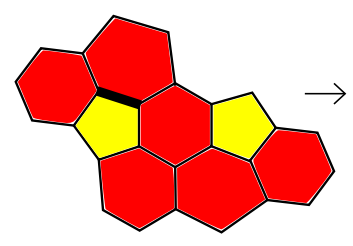

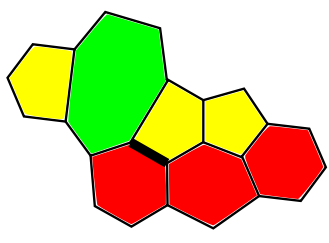

b)

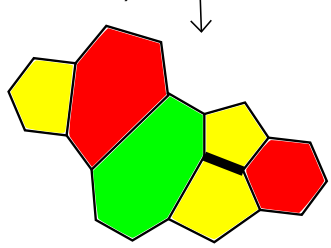

c)
Fig. 19 a) A patch on the fullerene; b)-d) it's reduction

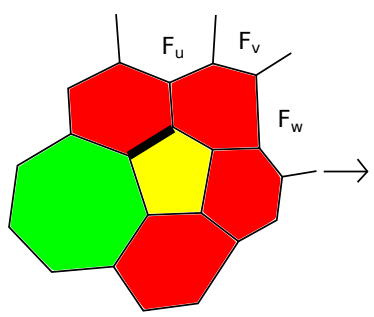

a)

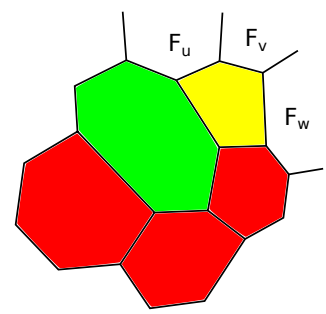

b)
Fig. 20 a) A patch on the polytope in $\mathscr{F}_{1}^{I P R}$, b) it's reduction

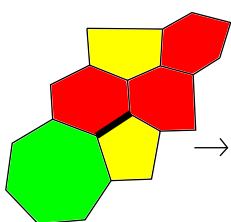

a)

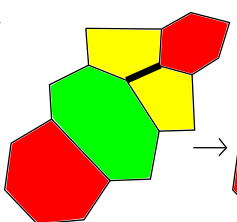

b)

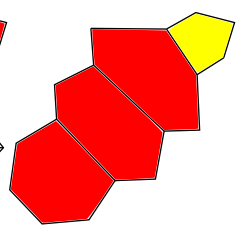

c)
Fig. 21 a) A patch on the polytope in $\mathscr{F}_{1}^{I P R}$, b)-c) it's reductions

Fig. 19 a). Then Fig. 19 b)-d) show how this patch can be reduced by straightening along edges. Then composition of the inverse operations gives operation $B_{3}$.

Case 3. $P$ has no hexagons adjacent to two pentagons. Then any pentagon is surrounded by a belt of hexagons intersecting no other pentagons. Then any straightening along common edge of a pentagon and a hexagon gives a polytope in $\mathscr{F}_{1}^{I P R}$ such that $P$ is obtained from it by operation $A_{6}$, which is a $(2,7 ; 5,5)$-truncation.

Now consider a polytope $P \in \mathscr{F}_{1}^{I P R}$ and a pentagon adjacent to the heptagon, as drawn on Fig. 20 a). Straightening the edge we obtain Fig. 20 b). Then $P$ is obtained from the resulting polytope $Q$ by operation $A_{7}$. The polytope $Q$ does not belong to $\mathscr{F}_{1}^{I R P}$ if and only if one of the facets $F_{u}, F_{v}$, or $F_{w}$ is a pentagon. If $F_{u}$ us a pentagon, then since $P$ has no 3and 4-belts, we obtain the patch drawn on Fig.21 a). Applying the straightenings as drawn on Fig. 21. b)-c), we obtain a fullerene $Q$ such that $P$ is obtained from $Q$ by operation $B_{2}$.

If $F_{v}$ is a pentagon, then since $P$ has no 3 - and 4-belts, we obtain the fragment drawn on Fig. 22 a). It is a patch if and 

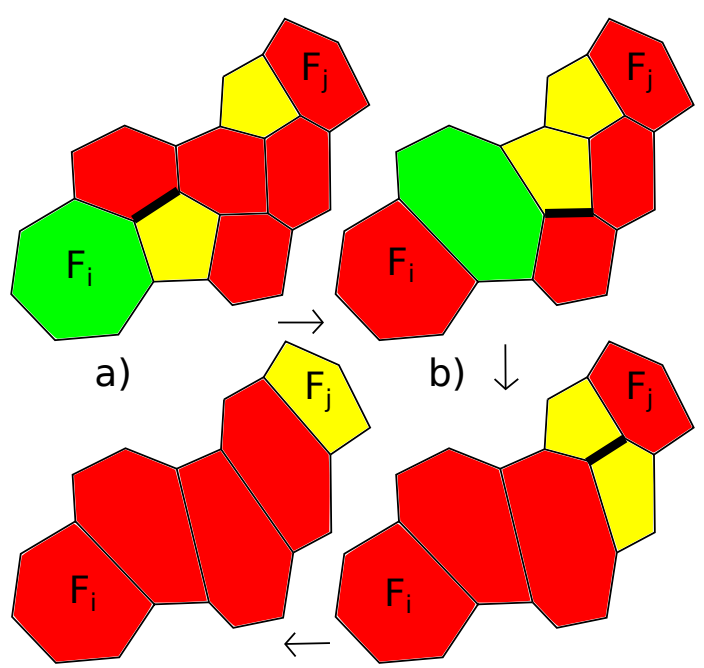

d)

C)

Fig. 22 a) A patch on the polytope in $\mathscr{F}_{1}^{I P R}$, b)-d) it's reductions

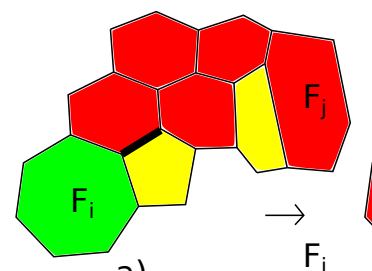

a)

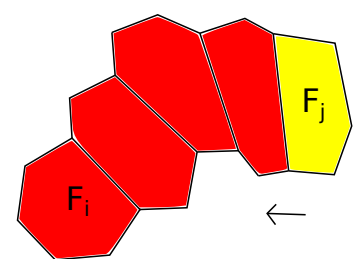

d)

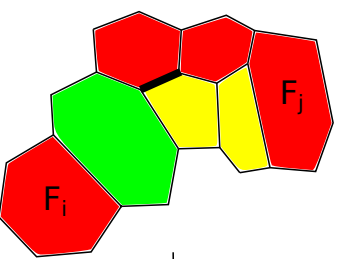

b) $\downarrow$

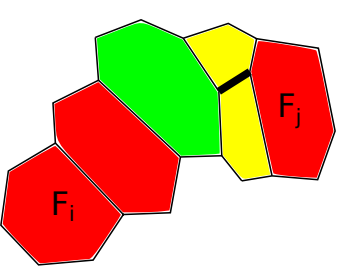

c)
Fig. 23 a) A patch on the polytope in $\mathscr{F}_{1}^{I P R}$, b)-d) it's reductions

only if $F_{i} \cap F_{j}=\varnothing$. Applying the straightenings as drawn on Fig. 22 b)-d), we obtain a fullerene $Q$. Theorem 7 implies that $F_{i} \cap F_{j}=\varnothing$; hence the same if true for $P$. Thus, we have a patch, and the polytope $P$ is obtained from $Q$ by operation $B_{4}$.

If $F_{v}$ is a hexagon and $F_{w}$ is a pentagon, then since $P$ has no 3- and 4-belts, we obtain the fragment drawn on Fig. 23 a). It is a patch if and only if $F_{i} \cap F_{j}=\varnothing$. Applying the straightenings as drawn on Fig. 23 b) -d), we obtain a fullerene $Q$. Theorem 7 implies that $F_{i} \cap F_{j}=\varnothing$ in $Q$; hence the same if true for $P$. Thus, we have a patch, and the polytope $P$ is obtained from $Q$ by operation $B_{5}$. This finishes the proof.

\section{Compliance with Ethical Standards}

Acknowledgements This work is supported by the Russian Science Foundation under grant 14-11-00414. The second author is the Young Russian Mathematics award winner.

Conflict of Interest: The authors declare that they have no conflict of interest.

\section{References}

1. Lord EA, Mackay AL, Ranganathan S (2006) New Geometries for New Materials. Cambridge University Press.

2. Grünbaum B (2003) Convex polytopes (2nd Edition). Graduate texts in Mathematics 221, Springer-Verlag, New York.

3. Ziegler GM (2007) Lectures on polytopes (7th Printing). Graduate texts in Mathematics, 152, Springer.

4. Curl RF (1996) Dawn of the Fullerenes: Experiment and Conjecture, Nobel Lecture.

5. Kroto H (1996) Symmetry, Space, Stars and $C_{60}$, Nobel Lecture.

6. Smalley RE (1996) Discovering the Fullerenes. Nobel Lecture.

7. Thurston WP (1998) Shapes of polyhedra and triangulations of the sphere. Geometry and Topology Monographs 1: 511-549.

8. Brinkmann G, Dress AWM (1997) A constructive enumeration of fullerenes. J Algorithms 23 (2): 345-358.

9. Brinkmann G, Goedgebeur J, McKay BD (2012) The generation of fullerenes. J Chem Inf Model 52: 2910-2918.

10. Brinkmann G, Coolsaet K, Goedgebeur J, Mélot H (2013) House of Graphs: a database of interesting graphs. Discrete Applied Mathematics 161: 311-314. Available at http://hog.grinvin.org

11. Brinkmann G, Franceus D, Fowler PW, Graver JE (2006) Growing fullerenes from seed: Growth transformations of fullerene polyhedra. Chem Phys Lett 428 (46): 386-393.

12. Hasheminezhad M, Fleischner H, McKay BD (2008) A universal set of growth operations for fullerenes. Chem Phys Lett 464: 118121.

13. Brinkmann G, Fowler PW (2003) A Catalogue of Growth Transformations of Fullerene Polyhedra. J Chem Inf Comp Sci 43: 8371843.

14. Yoshida M, Fowler PW (1997) Systematic relationships between fullerenes without spirals, Chem Phys Lett 278: 256-261.

15. Brinkmann G, Graver JE, Justus C (2009) Numbers of faces in disordered patches. J Math Chem 45(2): 263-278.

16. Andova V, Kardoš F, Škrekovski R (2016) Mathematical aspects of fullerenes. Ars Mathematica Contemporanea 11: 353-379.

17. Endo M, Kroto HW (1992) Formation of carbon nanofibers. J Phys Chem 96: 6941-6944.

18. Eberhard V (1891) Zur Morphologie der Polyheder. Leipzig.

19. Brückner M (1900) Vielecke und Vielflache. Leipzig.

20. Erokhovets NYu (2015) $k$-belts and edge cycles of simple 3polytopes with at most hexagonal facets (in russian). Far Eastern Mathematical Journal, 15(2): 197-213.

21. Došlić $T$ (1998) On lower bounds of number of perfect matchings in fullerene graphs. J Math Chem 24: 359-364.

22. Došlić T (2003) Cyclical edge-connectivity of fullerene graphs and $(k, 6)$-cages. J Math Chem 33(2): 103-112.

23. Kardoš F, Skrekovski R (2008) Cyclic edge-cuts in fullerene graphs. J Math Chem, 22: 121-132.

24. Kutnar K, Marušič D (2008) On cyclic edge-connectivity of fullerenes. Discrete Appl Math 156: 1661-1669.

25. Kardoš F, Krnc M, Lužar B, Skrekovski R (2010) Cyclic 7-edgecuts in fullerene graphs. J Math Chem 47(2): 771-789.

26. Buchstaber VM, Erokhovets NYu (2015) Graph-truncations of simple polytopes. P Steklov Inst Math 289:104-133. 
27. Buchstaber VM, Erokhovets NYu (2015) Construction of fullerenes. arXiv 1510.02948 .

28. Buchstaber VM, Erokhovets NYu (2016) Fullerenes, Polytopes and Toric Topology. Lecture Note Series, IMS, NUS, Singapore, arXiv: 1609.02949

29. Deza M, Dutour Sikirić M, Shtogrin MI (2013) Fullerenes and disk-fullerenes. Russ Math Surv+ 68(4): 665-720. 